\title{
Musculoskeletal Melioidosis in a Malaysian Return Driver with Chronic Osteomyelitis - A Rare Case Report
}

\author{
M. Jane Esther*, P.K. Rath and D. Thirumalaipandiyan \\ ${ }^{1}$ Microbiology, Doctors Diagnostic Centre, Trichy, India \\ ${ }^{2}$ Pathologist, Doctors diagnostic centre, Trichy, India \\ ${ }^{3}$ Orthopaedic surgeon, Thirumalai Ortho centre, Tanjore, India \\ *Corresponding author
}

\section{A B S T R A C T}

\section{Keywords \\ Musculoskeletal \\ melioidosis, Burkholderia \\ pseudomallei \\ osteomyelitis, Chronic \\ osteomyelitis \\ Article Info \\ Accepted: \\ 18 September 2018 \\ Available Online: \\ 10 October 2018}

Melioidosis is a systemic infectious disease caused by Burkholderia pseudomallei. It produces overlapping clinical manifestations which is indistinguishable from other diseases. Melioidosis of the bone and joint infections present chronically with relapses and recurrences. It is most of the time misdiagnosed as tuberculosis due to overlapping clinical, histopathological and radiological features. We have presented here a case report of an elderly Malaysian driver, who was a diabetic and presented with features of osteomyelitis. It was initially diagnosed as tubercular osteomyelitis. Microbial culture from relevant samples of the patient finally led to the diagnosis of musculoskeletal melioidosis of the hip joint. The clinical presentation, diagnosis, treatment and follow up details of the patient have been discussed as a case report.

\section{Introduction}

Melioidosis or Whitmore's disease is a systemic infectious disease which mimics many other diseases in clinical manifestations causing confusion in the diagnosis (Cheng and Currie, 2005). It can virtually affect any system presenting with pneumonia or pleural effusion, skin and soft tissue infection, genitourinary, bone and joint or central nervous system infection, chronic lymphadenopathy, abscesses, bacteraemia or sepsis (Cheng and Currie, 2005). It has a long incubation period which earned it the name "Vietnamese time bomb'. It is known for its chronicity because of the nature of the causative organism Burkholderia pseudomallei to grow and multiply intracellularly within the macrophages (Perumal Samy et al., 2017). The bacterium is intrinsically resistant to many antibiotics and requires prolonged treatment for cure and prevention of relapse (Perumal Samy et al., 2017). It is being sporadically reported. It is actually being reported where it is suspected. A high index of suspicion is necessary (Cheng and Currie, 2005). We have presented here a case report of a patient who was diagnosed as a case of musculoskeletal melioidosis by microbial culture, treated promptly and discharged successfully. It was decided to publish this case in detail to emphasize the importance of considering melioidosis in the differential diagnosis of systemic infectious diseases. 


\section{Case report}

A 55 year old male patient, who was a tractor driver in Malaysia, came to a hospital in India (Tanjavur district of Tamilnadu) with complaints of pain in his right hip joint since a week. His pain was dull aching in nature and became worse with movement. He had fever on and off that subsided with antipyretic medications. The patient gave a history of having suffered from a sub cutaneous abscess before four weeks, in the right gluteal region while he was in Malaysia. It was treated with incision and drainage and a course of antibiotics. After treatment for about 10 days, the wound healed and the patient was apparently normal.

Four weeks later, he presented with pain in the right hip joint (present visit to the clinician). On general examination, the patient was normally built, not anaemic/ cyanosed, his blood pressure was 130/80 mm Hg, pulse rate was 94/minute, cardiovascular system, respiratory system and gastrointestinal system were apparently normal.

On examination of the hip joint, there were erythema, tenderness and reduced range of movements over the right hip. His ESR and CRP were elevated. Based on his history, clinical features and laboratory findings, septic arthritis of the hip joint was suspected and the patient was started on ciprofloxacin $500 \mathrm{mg}$ 12 hourly. The patient was a known case of diabetes mellitus since three years, on treatment.

MRI of the hip joint showed hyper intense changes involving the right femoral neck, trochanter, proximal shaft, head and marrow with moderate effusion. There was also a significant inflammation and edema of the peri-articular soft tissues involving the adductor and hamstring muscles of the thigh as shown in figure 1 .
Surgical opening of the hip joint was done. Frank pus was found in the acetabulum of the hip. Osteomyelitis of the proximal end of femur was suspected. Curettings from acetabulum was sent for histopathological examination and wound swab for microbiological examination.

Histopathological examination showed bone with surrounding fibrovascular tissue densely infiltrated by polymorphs. Caseation necrosis, epithelioid giant cells and Langerhans giant cells were also seen. There was bony destruction. Microbiological culture of wound swab showed no growth. With all these findings a diagnosis of tubercular osteomyelitis of right proximal femur was made. The patient was started on ATT (Anti tubercular therapy) and it was continued for 2 months. After 2 months the patient presented again to the OPD with a sinus at the surgical site with persistent discharge for more than a month.

The hip joint was opened again. Through iliofemoral approach, the proximal femur was exposed. The sequestrum that was over the cavity was enlarged and saucerisation was done. The sequestrum was sent for microbiological culture and sensitivity testing. Direct microscopic examination of the sample by gram staining showed many pus cells and gram negative bacilli. The sample was inoculated onto blood agar and mac conkey agar. After 24 hours incubation, blood agar showed smooth, grey and translucent colonies and MacConkey agar showed light pink glistening colonies as shown in figure $2 \mathrm{a}$.

Gram staining of the bacterial colony from MacConkey agar showed gram negative bacilli with safety pin appearance as shown in figure $2 \mathrm{~b}$. The organism was oxidase positive, motile, non-fermenter of mannitol, alkaline slat with no change in butt of triple sugar iron agar media, did not utilise citrate and was also 
a non-producer of indole. Based on these findings the organism was presumptively identified as Burkholderia pseudomallei and its identity was confirmed with Vitek 2 compact automated system. Antibiogram was done. The organism was found to be susceptible to all the drugs that were tested by Kirby Bauer disk diffusion technique, which includes Amoxycillin clavulanate, Piperacillin tazobactam, Chloramphenicol, Cotrimoxazole, Ciprofloxacin, Ofloxacin, Imipenem, Meropenem and Doxycycline. Finally, the diagnosis of Musculoskeletal melioidosis of the right hip joint was made.

ATT was stopped. The patient was treated with intravenous injections which included traxol S (Ceftriaxone-sulbactam) 1.5 gram 12 hourly, ciprofloxacin $500 \mathrm{mg} 12$ hourly, piperacillin-tazobactam $4.5 \mathrm{~g} 12$ hourly for two weeks. He responded well clinically.

The wound healed and he was discharged in two weeks with oral ciprofloxacin $500 \mathrm{mg}$ $\mathrm{BD}$, doxycycline $100 \mathrm{mg} \mathrm{BD}$ and cotrimoxazole $960 \mathrm{mg} \mathrm{BD}$. He was advised to continue the oral antibiotic therapy for six months. He is being followed up once in two weeks. The patient is doing well at present.

\section{Results and Discussion}

Melioidosis is an emerging life threatening infectious disease caused by Burkholderia pseudomallei, which is a non-fermenting gram negative bacillus. This bacterium is mostly seen as a saprophyte in the tropical regions of the world (Raja et al., 2016). It is present in the soil and water and enters through abraded skin or by inhalation. Sexual transmission and transmission through breast milk have been reported (Cheng and Currie, 2005). The disease is not zoonotic. Human to human transmission can occur via respiratory droplets or secretions of the infected individuals (Raja et al., 2016). It is a category B bioterrorism agent and requires BSL-3 precautions in the laboratory. It is prevalent in the tropical countries like Southeast Asia and Northern Australia (Vivek et al., 2010). However, international travel has taken this disease outside these countries to many other parts of the world (Raja et al., 2016). Cases have been reported from Malaysia, Pakistan, Indonesia, Sri Lanka and Japan. Our patient is a resident of Malaysia, who has travelled to India, which is a strong risk factor for acquiring melioidois.

Immunosuppressive conditions like diabetes mellitus, HIV, cancer, chronic liver, renal or lung diseases can predispose to melioidosis (Raja et al., 2016). Diabetes mellitus is the immunocompromised state associated with our patient. Our patient is a driver by occupation, who is more prone to acquire cuts/ abrasions and also for exposure to contaminated soils (Perumal Samy et al., 2017).

Melioidosis has varied clinical manifestations overlapping with other diseases making it a challenging task to diagnose it accurately (Raja et al., 2016). It can present as a localized infection with ulcer or abscess, pulmonary infection with respiratory symptoms, blood stream infections with septicaemic manifestations or disseminated infection with hyperpyrexia, anorexia, myalgia, bone and joint infection or seizures due to infection of the central nervous system (Raja et al., 2016). It most of the time presents as chronic infection with recurrences, as in our patient (Perumal Samy et al., 2017).

Bone and joint infections due to $B$. pseudomallei can manifest as localised abscess formation, septicaemia, septic arthritis, chronic or recurrent osteomyelitis, which are similar to the manifestations of Staphylococcal or tubercular infections (Harris et al., 2017). Knee joint is one that is most commonly affected in melioidosis, followed 
by the hip joint (Raja et al., 2016). Burkholderia pseudomallei infection is most of the time confused with tuberculosis due to overlapping clinical, histopathological and radiological features, as it was in our patient (Perumal Samy et al., 2017). So
B. pseudomallei infection must be considered in the differential diagnoses of any patient with bone and joint infection. Early diagnosis can be made only through clinical suspicion and laboratory confirmation. Treatment at an early stage can save the patient's life.
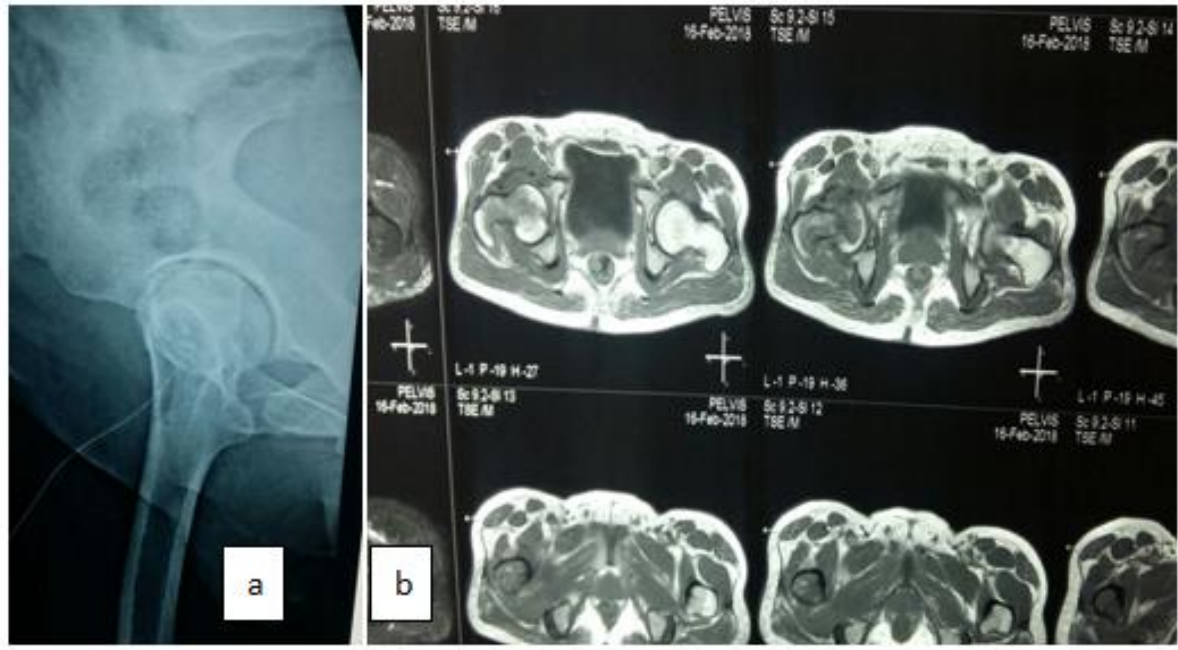

Figure1. X-ray (a) and MRI scan (b) of the right hip joint showing features of chronic osteomyelitis.
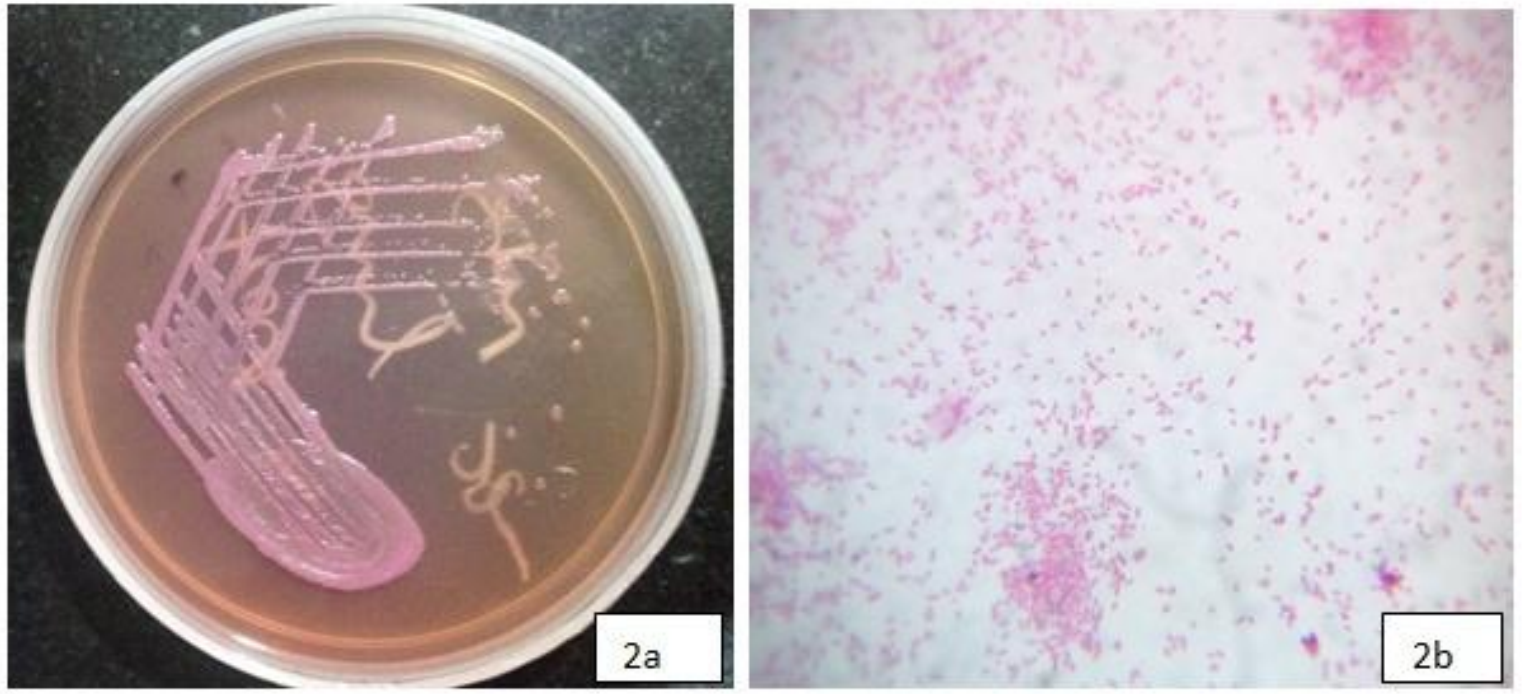

Figure 2.a) Light pink coloured colonies of Burkholderia pseudomallei on Macconkey agar. b) Burkholderia pseudomallei colony gram stain showing gram negative bacilli with bipolar staining. 
The only way for correct diagnosis of melioidosis is microbiological culture. Appropriate sample is the corner stone for a positive culture report (Raja et al., 2016). Aspirated pus from abscesses, bone in case of osteomyelitis, tissues from deeper parts of the wounds or ulcers can yields positive cultures. Swabbing from the superficial parts of the wounds or lesions of patients has very poor chances of recovery of any organism in culture (Raja et al., 2016). This could have been the reason for the initial negative culture report in our patient. Transportation of the sample under appropriate conditions is also very important. Negative cultures in the presence of infection can also occur if antimicrobials were administered to the patient prior to sample collection (Harris et al., 2017). At any case, samples must be collected for microbial culture before administering the first dose of antibiotic to the patient (Harris et al., 2017). Modified Ashdown media is selective for the isolation of B. pseudomallei (Cheng et al., 2006). However, it can be identified even on routine culture media like MacConkey agar if the microbiologist is suspicious of the organism (Harris et al., 2017).

Musculoskeletal melioidosis must be treated by extensive debridement of the infected bones/ tissues followed by prolonged antibiotic therapy. $B$. pseudomallei is inherently resistant to penicillins, aminoglycosides, first and second generation cephalosporins (Raja et al., 2016). Deep seated infections require intravenous treatment with ceftazidime $(50 \mathrm{mg} / \mathrm{kg} /$ day 8 hourly or $100 \mathrm{mg} / \mathrm{kg} /$ day in severe melioidosis $)$, meropenem $(25 \mathrm{mg} / \mathrm{kg} \quad 8$ hourly), cotrimoxazole (40-60 mg/kg/day 12 hourly) and ciprofloxacin (500 mg 12 hourly) in the acute phase for 2 months, followed by any two of cotrimoxazole $(2.96 \mathrm{~g}$ tablet every 12 hourly), doxycycline (100 mg tablet 12 hourly) and amoxicillin-clavulanate $(625 \mathrm{mg}$
8 hourly) in the eradication phase for atleast 6 months in osteomyelitis or 3 months in septic arthritis.[Raja et al., 2016). It is absolutely necessary for the patient to complete the eradication therapy and for the clinician to regularly follow up the patient's compliance to treatment because of a higher relapse rate associated with $B$. pseudomallei infection (30\% relapse if the patient is treated with antibiotics for less than 8 weeks as against less than $10 \%$ with completed treatment) (Raja et al., 2016).

An incidence of around 7.6 and 27 percent of musculoskeletal Melioidosis has been reported from Australia and Thailand respectively as against sporadic cases being reported in India (Raja et al., 2016). Melioidosis is being under reported in India because of lack of awareness about the disease, lack of clinical suspicion and nonspecific clinical manifestations of the disease (Raja et al., 2016).

Musculoskeletal Melioidosis is clinically indistinguishable from bone and joint infections caused by other bacteria and from tuberculosis. A high index of suspicion of melioidosis is necessary, both by the clinician and the clinical microbiologist. Melioidosis must be kept in mind as a differential diagnosis of infectious disease involving any system of the patient, especially in patients who travel frequently and in those who return from tropical or subtropical regions (Perumal Samy et al., 2017). Though the empirical antibiotics that are commonly used are effective against $B$. pseudomallei, a longer duration of treatment is needed to eradicate the organism. So melioidosis cannot be effectively treated unless exactly diagnosed. Reporting of non-fermenting gram negative bacilli must be done with caution. Reporting plainly as "NFGNB isolated in culture" might miss the diagnosis of a dangerous organism like B.pseudomallei, which if treated at the 
right time could save the life of the patient. Efforts must be made to identify the exact bacterium that is isolated. Automated systems like Vitek can help in early accurate diagnosis. Clinicians must be made aware of the right way of collecting and transporting the right specimens for microbiological culture so that early diagnosis can be made. Clinical and microbiological suspicion, accurate identification and the right treatment for the right duration can save patients with melioidosis.

\section{References}

Cheng AC, and Currie BJ. Melioidosis: epidemiology, pathophysiology, and management. Clinical Microbiology Reviews. 2005; 18: 383-416.

Cheng AC, Wuthiekanun V, Limmathurosakul D, Wongsuvan G, Day NPJ, Peacock SJ. Role of selective and nonselective media for isolation of Burkholderia pseudomallei from throat swabs of patients with melioidosis.
Journal of Clinical Microbiology. 2006; 44: 2316.

Harris AM, Bramley AM, Jain S, Arnold SR, Ampofo K, Self WH et al., influence of antibiotics on the detection of bacteria by culture-based and cultureindependent diagnostic tests in patients hospitalized with community-acquired pneumonia. Open Forum Infectious Diseases. 2017; 4

Perumal Samy R, Stiles BG, Sethi G, Lim LHK; 2017. Melioidosis: Clinical impact and public health threat in the tropics. PLoS Negl Trop Dis., 11: e0004738.

Raja NS, Scarsbrook C. Burkholderia Pseudomallei Causing Bone and Joint Infections: A Clinical Update. Infectious Diseases and Therapy. 2016; 5: 17-29.

Vivek P, Sripathi P R, Sugandhi R, Kiran K.V A, Singh C S; Burkholderia pseudomallei musculoskeletal infections (melioidosis) in India; Indian Journal of Orthopaedics; 2010; 44: 216-220.

\section{How to cite this article:}

Jane Esther, M., P.K. Rath and Thirumalaipandiyan, D. 2018. Musculoskeletal Melioidosis in a Malaysian Return Driver with Chronic Osteomyelitis - A Rare Case Report. Int.J.Curr.Microbiol.App.Sci. 7(10): 2264-2269. doi: https://doi.org/10.20546/ijcmas.2018.710.261 